\title{
Two cases of thyroid gland invasion by upper mediastinal carcinoma
}

\author{
Haruhiko Yamazaki', Hiroyuki Iwasaki', Yoichiro Okubo², Nobuyasu Suganuma', \\ Katsuhiko Masudo ${ }^{3}$, Hirotaka Nakayama4, Yasushi Rino ${ }^{4}$ and Munetaka Masuda 4 \\ 1Department of Breast and Endocrine Surgery, 2Department of Pathology, Kanagawa Cancer Center, Yokohama, \\ Japan, ${ }^{3}$ Department of Breast and Thyroid Surgery, Yokohama City University Medical Center, Yokohama, Japan, and \\ ${ }^{4}$ Department of Surgery, Yokohama City University School of Medicine, Yokohama, Japan
}

Correspondence

should be addressed

to $\mathrm{H}$ Yamazaki

Email

paruo0413@gmail.com

\section{Summary}

The objective this study is to report two cases of thyroid gland invasion by upper mediastinal carcinoma. Mediastinal tumors are uncommon and represent $3 \%$ of the tumors seen within the chest. In reports on mediastinal masses, the incidence of malignant lesions ranged from 25 to $49 \%$. The thyroid gland can be directly invaded by surrounding organ cancers. We report these cases contrasting them to the case of a thyroid cancer with mediastinal lesions. Case 1 was a 73-year-old woman who was diagnosed with papillary thyroid carcinoma, and she underwent surgery and postoperative radioactive iodine. Case 2 was a 74-year-old man who was diagnosed with non-small-cell lung carcinoma, favor squamous cell carcinoma, and he underwent chemoradiotherapy. Case 3 was a 77-year-old man who was diagnosed a thymic carcinoma based on pathological findings and referred the patient to thoracic surgeons for surgical management. The images of the three cases were similar, and the differential diagnoses were difficult and required pathological examination. Primary thyroid carcinoma and invading carcinoma originating from the adjacent organs need to be distinguished because their prognoses and treatment strategies are different. It is important to properly diagnose them by images and pathological findings.

\section{Learning points:}

- The thyroid gland in the anterior neck can be directly invaded by surrounding organ cancers.

- Primary thyroid carcinoma and invading carcinoma originating from the adjacent organs need to be distinguished because their prognoses and treatment strategies are different.

- It is important to properly diagnose by images and pathological findings.

\section{Background}

The thyroid gland in the anterior neck can be directly invaded by surrounding organ cancers, and laryngeal and hypopharyngeal carcinomas often invade the gland. However, reports of other carcinomas directly invading the thyroid gland are few (1). We encountered two patients with mediastinal malignant tumors that directly invaded the thyroid gland and required differentiation from primary thyroid cancer. We report these cases contrasting them to the case of a thyroid cancer with mediastinal lesions.

\section{Case reports}

\section{Case 1}

\section{Case presentation}

A 73-year-old woman consulted a nearby physician due to bloody sputum. A bronchoscope examination revealed a tumor projecting into the lumen and she was diagnosed as having a papillary thyroid carcinoma (PTC) after a biopsy. She visited our hospital for treatment. 


\section{Investigation}

The blood test results included a thyroid-stimulating hormone (TSH) at $2.62 \mu \mathrm{IU} / \mathrm{mL}$, free triiodothyronine (F-T3) at $3.07 \mathrm{pg} / \mathrm{mL}$, free thyroxine (F-T4) at $1.04 \mathrm{ng} /$ $\mathrm{mL}$, thyroglobulin $(\mathrm{Tg})$ at $609 \mathrm{ng} / \mathrm{mL}$ and thyroglobulin antibody (TgAb) at $361 \mathrm{IU} / \mathrm{mL}$. Ultrasonography (US) revealed a low echoic irregular mass with unclear borders in the lower pole of the right lobe of thyroid (Fig. 1A). Computed tomography (CT) revealed an irregular tumor measuring $3.7 \mathrm{~cm}$ on the right side of the trachea with a protrusion into the tracheal lumen (Fig. 1B). No continuity between the tumor and the thyroid was apparent (Fig. 1C). She had no distant metastases. Histological sections of the previous bronchoscopy revealed a papillary growth. Immunohistochemistry (IHC) results were positive for thyroid transcription factor 1 (TTF-1), Tg and PAX-8. The lesion was diagnosed as a tracheal invasion of mediastinal lymph node metastasis due to PTC (UICC 8th edition, T1bN1aM0 StageII).

\section{Treatment}

The patient underwent total thyroidectomy, central neck and upper mediastinal dissections and tracheal and right recurrent nerve combined resections. The postoperative course was uneventful, and we discharged the patient on the 12th postoperative day. Histopathological finding confirmed the diagnosis of PTC. The tumor formed a papillary structure, and individual cancer cells had nuclear grooves (Fig. 1D and E). We ordered postoperative radioactive iodine (RAI).

\section{Outcome and follow-up}

The patient has no evidence of recurrence 8 months after operation.

\section{Case 2}

\section{Case presentation}

A 74-year-old man consulted his physician due to bloody sputum and hoarseness. CT revealed an upper mediastinal tumor projecting into the bronchial lumen. Biopsy through bronchoscopy diagnosed a poorly differentiated carcinoma. IHC results were partially positive for CK7 and TTF-1 (Fig. 2E), and negative for CK20, chromogranin A, synaptophysin and CD56. The tumor was suspected to be a tracheal invasion from a thyroid cancer, and the patient was referred to our hospital.
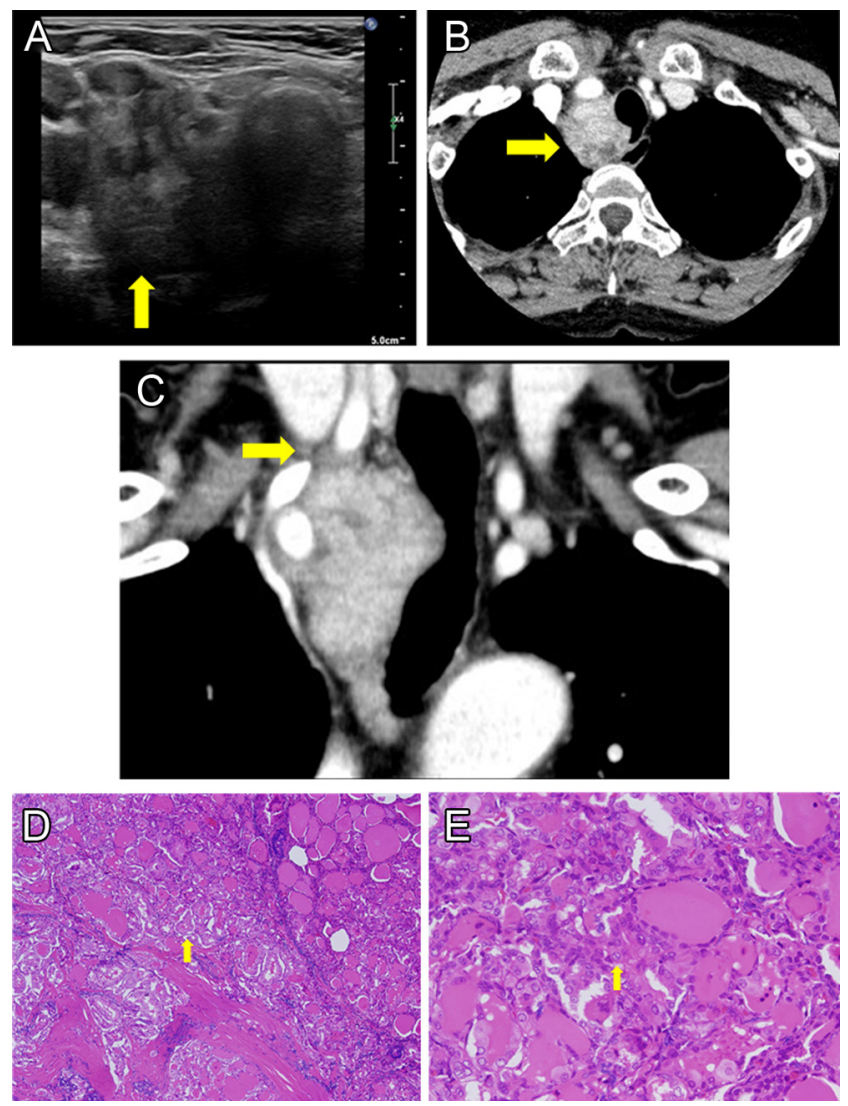

\section{Figure 1}

Case of papillary thyroid carcinoma. (A) Ultrasonography revealed a low echoic irregular mass with unclear borders in the lower pole of the right lobe of thyroid (arrow). (B) Computed tomography revealed an irregular tumor measuring $3.7 \mathrm{~cm}$ on the right side of the trachea with a protrusion into the tracheal lumen (arrow). (C) No continuity between the tumor and the thyroid was apparent (arrow). (D and E) Histologic sections of the resection specimen showed that the tumor formed a papillary structure, and individual cancer cells had nuclear grooves (arrows). (D) Original magnification $\times 100$, (E) Original magnification $\times 400$.

\section{Investigation}

The blood tests on admission showed a TSH at $3.19 \mu \mathrm{IU} / \mathrm{mL}, \mathrm{F}-\mathrm{T} 3$ at $1.97 \mathrm{pg} / \mathrm{mL}, \mathrm{F}-\mathrm{T} 4$ at $0.86 \mathrm{ng} / \mathrm{mL}$, $\mathrm{Tg}$ at $32.3 \mathrm{ng} / \mathrm{mL}, \mathrm{TgAb}$ at $642 \mathrm{IU} / \mathrm{mL}$, CEA at $3.4 \mathrm{ng} / \mathrm{mL}$ (normal upper limit (NUL): $5.0 \mathrm{ng} / \mathrm{mL}$ ), squamous cell carcinoma (SCC) antigen at $2.7 \mathrm{ng} / \mathrm{mL}$ (NUL: $1.5 \mathrm{ng} / \mathrm{mL}$ ), CYFRA at $<1.0 \mathrm{ng} / \mathrm{mL}$ (NUL: $3.5 \mathrm{ng} / \mathrm{mL}$ ) and ProGRP at $59.0 \mathrm{pg} / \mathrm{mL}$ (NUL: $81.0 \mathrm{pg} / \mathrm{mL}$ ). US revealed an irregular low echoic area in contact with the lower pole of the right lobe of thyroid (Fig. 2A). CT revealed an irregular tumor measuring $8 \mathrm{~cm}$ on the right side of the trachea protruding into the tracheal lumen (Fig. 2B). The head side of the tumor was in contact with the lower pole of the right lobe of thyroid (Fig. 2C). There was no cervical lymph node metastasis. He had no distant metastases. Transbronchial biopsy specimen was revised. 

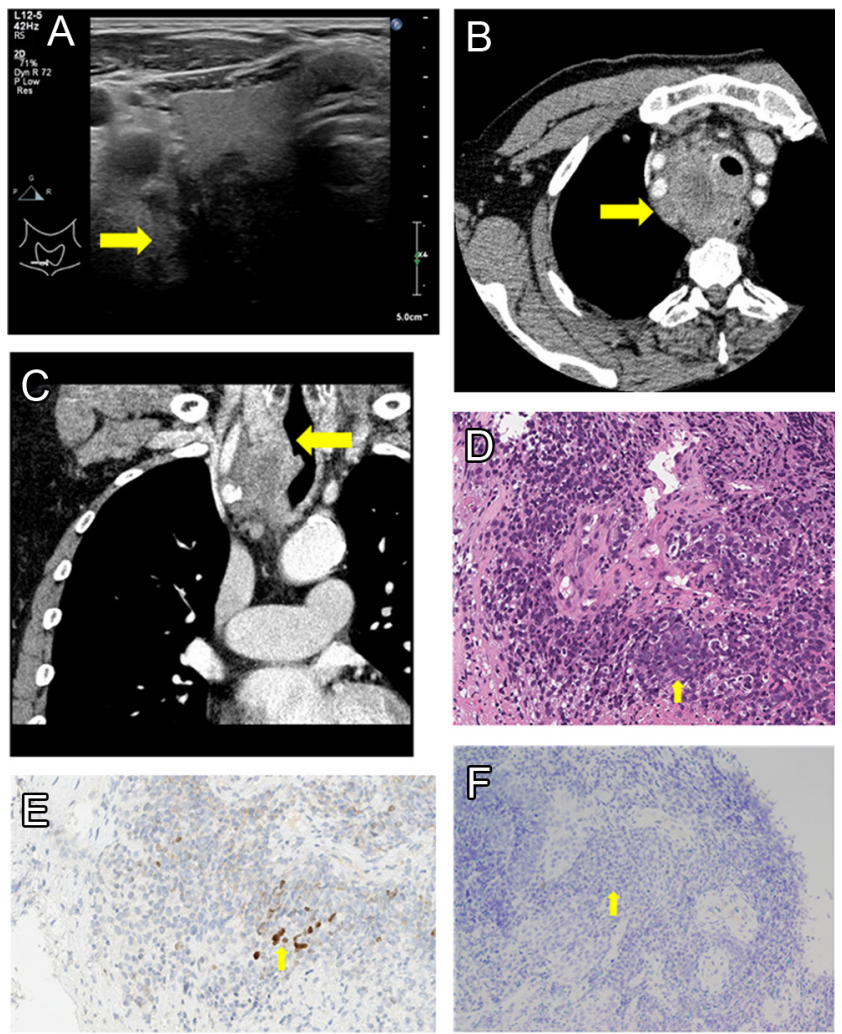

Figure 2

Case of mediastinal type non-small-cell lung cancer. (A) Ultrasonography revealed an irregular low echoic area in contact with the lower pole of the right lobe of thyroid (arrow). (B) Computed tomography revealed an irregular tumor measuring $8 \mathrm{~cm}$ on the right side of the trachea protruding into the tracheal lumen (arrow). (C) The head side of the tumor was in contact with the lower pole of the right lobe of thyroid (arrow). (D) Histologic sections of the biopsy through bronchoscopy showed that the tumor cell had high nuclear-plasmic ratio (arrow). Original magnification $\times 200$. (E and F) Immunohistochemistry revealed partially positive for thyroid transcription factor 1 (E) (arrow) and negative for PAX-8 (F) (arrow). Original magnification $\times 200$.

The tumor cell had high nuclear-plasmic ratio and poorly differentiated SCC was diagnosed (Fig. 2D). Because the main lesion was within the mediastinum, differential diagnosis included thyroid, lung or esophageal cancer. The later was excluded by the upper gastrointestinal endoscopy. We performed additional IHC observing diffuse p40 and partially CAM5.2 positivity, and CD5, nuclear protein in testis (NUT), PAX-8 (Fig. 2F) and Tg negativity.

\section{Outcome and follow-up}

We discarded SCC of the thyroid gland, and we diagnosed the patient as having non-small-cell lung carcinoma, squamous cell carcinoma type (UICC 8th edition, T4N3M0 StageIIIC) and he underwent chemoradiotherapy by pulmonologists.

\section{Case 3}

\section{Case presentation}

A 77-year-old man consulted a nearby physician due to cervical discomfort. CT revealed an upper mediastinal tumor that was diagnosed as a mediastinal goiter. The patient was referred to us.

\section{Investigation}

The blood test showed a TSH at $1.86 \mu \mathrm{IU} / \mathrm{mL}$, F-T3 at $1.64 \mathrm{pg} / \mathrm{mL}, \quad F-T 4$ at $1.28 \mathrm{ng} / \mathrm{mL}, \mathrm{Tg}$ at $26.8 \mathrm{ng} / \mathrm{mL}$, $\mathrm{TgAb}$ at $<10 \mathrm{IU} / \mathrm{mL}$, CEA at $4.6 \mathrm{ng} / \mathrm{mL}$, SCC antigen at $1.0 \mathrm{ng} / \mathrm{mL}$, CYFRA at $2.4 \mathrm{ng} / \mathrm{mL}$ and ProGRP at $81.6 \mathrm{pg} /$ $\mathrm{mL}$. US revealed a low echoic tumor with unclear borders in the lower pole of the left lobe of thyroid (Fig. 3A). CT revealed an irregular tumor measuring $7.5 \mathrm{~cm}$. The tumor was developing from the upper mediastinum to the lower pole of the left lobe of thyroid (Fig. 3B and C). The tumor invaded brachiocephalic vein, and there was no cervical
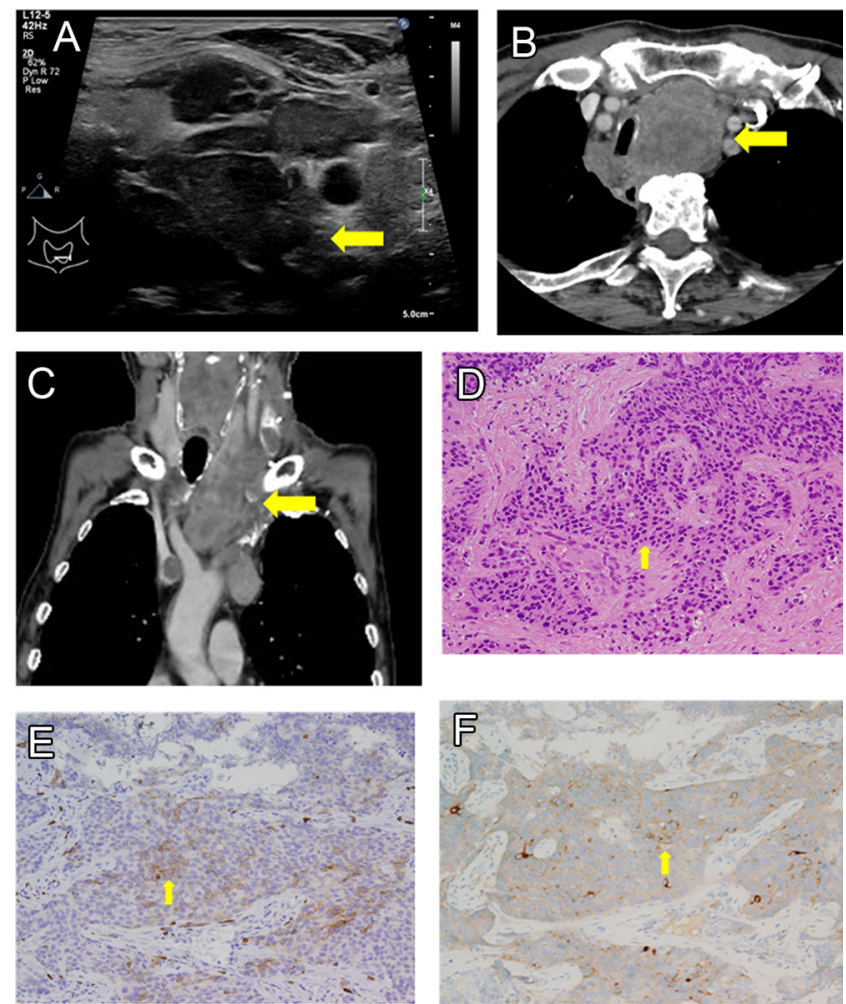

\section{Figure 3}

Case of thymic carcinoma. (A) Ultrasonography revealed a low echoic tumor with unclear borders in the lower pole of the left lobe of thyroid (arrow). (B and C) Computed tomography revealed an irregular tumor measuring $7.5 \mathrm{~cm}$ (arrow). The tumor was developing from the upper mediastinum to the lower pole of the left lobe of thyroid (arrow). (D) Histologic sections of the core needle biopsy showed that the tumor contained many nuclear fissions and included necrosis (arrow). Original magnification $\times 200$. ( $E$ and $F$ ) Immunohistochemistry revealed positive for CD5 (E) and synaptophysin (F) (arrows). Original magnification $\times 200$. 
lymph node metastasis. He had no distant metastases. We suspected SCC based on the tumor cytology. The core needle biopsy was performed to identify the primary organ. The tumor contained many nuclear fissions and included necrosis (Fig. 3D). IHC results were positive for CD5 (Fig. 3E), chromogranin A and synaptophysin (Fig. 3F) and negative for c-kit, p40 and TTF-1. We suspected thymus-derived cancer from the IHC results.

\section{Outcome and follow-up}

We diagnosed the patient as having a thymic carcinoma (UICC 8th edition, T3N2M0 StageIVB) based on pathological findings and referred the patient to thoracic surgeons, and he will undergo surgical management.

\section{Discussion}

Mediastinal tumors are uncommon and represent 3\% of the tumors seen within the chest. In reports on mediastinal masses, the incidence of malignant lesions ranged from 25 to $49 \%$ (2). Significant advances in the diagnosis of mediastinal lesions have been developed including through imaging, biopsy and immunohistochemical techniques. We encountered two patients with SCC invading the thyroid gland and one with PTC. The images of the three cases were similar, and the differential diagnoses were difficult and required pathological examination. However, confirmation of the diagnosis by cytology or core needle biopsy is often difficult (3).

Case 1 had a tumor with mediastinal tracheal invasion. Three major immunohistochemical markers differentiate thyroid cancers: PAX-8, Tg and TTF-1. The bronchoscopy specimen showed that these three markers were positive for papillary tumor cells, and the patient was diagnosed as having a tracheal invasion by a PTC. Surgery is the first choice for the initial treatment of differentiated thyroid cancers (DTCs). And postoperative RAI has been associated with improved prognosis. Our case had a tracheal invasion with a high risk of recurrence. Tyrosine kinase inhibitors (TKIs) are effective for RAI-refractory DTC. We will consider using a TKI if recurrence occurs.

Case 2 also had a tumor with a mediastinal tracheal invasion. The bronchoscopy specimen showed a poorly differentiated SCC, because p40 was diffusely positive. Tg and TTF-1 do not always become positive in IHC samples of primary thyroid SCCs (4). Therefore, Tg and TTF-1 were not useful for distinguishing between primary thyroid SCC and SCC of the lung. Suzuki et al. reported that the positive rates of PAX-8 in primary thyroid SCCs is $90.9 \%$ and that PAX-8 staining could help differentiate between primary thyroid SCC and invasion or metastasis from extrathyroidal SCCs (3). We also had to exclude anaplastic thyroid carcinoma (ATC). Bishop et al. reported that the tissue specificity of PAX8 expression might be useful in resolving the differential diagnosis of ATC such as the distinction between its squamoid variant and SCC of the head and neck (5). Therefore, PAX8 staining is an excellent marker for carcinomas of follicular epithelial origin. Case 2 was negative for Tg and PAX-8, and the image findings were not typical of thyroid cancer. Therefore, we diagnosed the patient as having SCC of the lung. We chose chemoradiotherapy for our patient. Some tissue types of lung cancer present high sensitivity to chemotherapy. On the other hand, the effectiveness of chemotherapy for thyroid cancer is uncertain, and the distinction between thyroid and lung cancers is important.

Case 3 had a low echoic tumor with unclear borders in the lower pole of the left lobe of the thyroid gland in US images and was a suspected SCC by cytology. We performed core needle biopsy to identify the primary organ. IHC results were positive for CD5, chromogranin $A$ and synaptophysin. Large cell neuroendocrine carcinoma (LCNEC) was suspected from tissue morphology. However, CD5 was positive and thymusderived cancer was considered first. We had to distinguish between a thymic carcinoma and a carcinoma showing thymus-like differentiation (CASTLE). These markers cannot help differentiate between thymic carcinomas and CASTLE (6). Considering the image findings, we diagnosed the tumor as a thyroid invasion of thymic carcinoma because the main lesion was within the mediastinum. Thymic carcinoma is rare and comprises only $0.06 \%$ of thymic neoplasms (7). Its prognosis is poor with an overall 5-year survival of $60 \%$ and 10 -year survival of $40 \%$. Curative surgery and postoperative radiotherapy contribute to the improvement of progression-free survival and overall survival. If surgery is difficult, chemotherapy may be done (8). On the other hand, the prognosis of CASTLE is relatively good, and its 5-year and 10-year cause-specific survival rates are 90 and $82 \%$, respectively (9).

The effectiveness of thyroidectomy for thyroid invasions by cancers originating from adjacent organs is unclear. The frequency of neoplastic involvement of the thyroid in advanced SCC of the larynx varies between 1 and 30\% (1). Lobectomy or total thyroidectomy should be selected according to the metastatic lesion site for thyroid metastasis by other organ cancers (10). In case 3, only the left lobe of the gland was invaded. Therefore, 
the left lobectomy at the same time as resection of the thymic carcinoma was appropriate.

\section{Conclusion}

These three cases had major lesions in the mediastinum, but the diagnosed carcinomas were different. Because each cancer has different treatment strategies and prognoses, it is important to properly diagnose them by images and pathological findings.

\section{Declaration of interest}

The authors declare that there is no conflict of interest that could be perceived as prejudicing the impartiality of the research reported.

\section{Funding}

This research did not receive any specific grant from any funding agency in the public, commercial or not-for-profit sector.

\section{Patient consent}

Written informed consent was obtained from the patients for publication of this case report and any accompanying images.

\section{Author contribution statement}

$\mathrm{H}$ Y prepared the manuscript. H I, N S, K M and $\mathrm{H} \mathrm{N}$ performed the surgery and cared for the patient. Y O performed the pathologic examination. Y R and $\mathrm{M} \mathrm{M}$ comprehensively supervised this case report. All authors read and approved the final manuscript.

\section{Acknowledgments}

The authors thank Enago (https://www.enago.jp/) for editing a draft of this manuscript.

\section{References}

1 Mangussi-Gomes J, Danelon-Leonhardt F, Moussalem GF, Ahumada NG, Oliveira CL \& Hojaij FC. Thyroid gland invasion in advanced squamous cell carcinoma of the larynx and hypopharynx. Brazilian Journal of Otorhinolaryngology 201783 269-275. (https://doi. org/10.1016/j.bjorl.2016.03.019)

2 Aroor AR, Prakasha SR, Seshadri ST \& Raghuraj U. A study of clinical characteristics of mediastinal mass. Journal of Clinical and Diagnostic Research 20148 77-80. (https://doi.org/10.7860/ JCDR/2014/7622.4013)

3 Suzuki A, Hirokawa M, Takada N, Higuchi M, Yamao N, Kuma S, Daa T \& Miyauchi A. Diagnostic significance of PAX8 in thyroid squamous cell carcinoma. Endocrine Journal 201562 991-995. (https://doi.org/10.1507/endocri.EJ15-0226)

4 Fassan M, Pennelli G, Pelizzo MR \& Rugge M. Primary squamous cell carcinoma of the thyroid: immunohistochemical profile and literature review. Tumori 200793 518-521. (https://doi. org/10.1177/030089160709300522)

5 Bishop JA, Sharma R \& Westra WH. PAX8 immunostaining of anaplastic thyroid carcinoma: a reliable means of discerning thyroid origin for undifferentiated tumors of the head and neck. Human Pathology 201142 1873-1877. (https://doi.org/10.1016/j. humpath.2011.02.004)

6 Ge W, Yao YZ, Chen G \& Ding YT. Clinical analysis of 82 cases of carcinoma showing thymus-like differentiation of the thyroid. Oncology Letters 201611 1321-1326. (https://doi.org/10.3892/ ol.2015.4055)

7 Greene MA \& Malias MA. Aggressive multimodality treatment of invasive thymic carcinoma. Journal of Thoracic and Cardiovascular Surgery 2003125 434-436. (https://doi.org/10.1067/mtc.2003.133)

8 Ahmad U, Yao X, Detterbeck F, Huang J, Antonicelli A, Filosso PL, Ruffini E, Travis W, Jones DR, Zhan Y, et al. Thymic carcinoma outcomes and prognosis: results of an international analysis. Journal of Thoracic and Cardiovascular Surgery 2015149 95-100, 101.e1. (https://doi.org/10.1016/j.jtcvs.2014.09.124)

9 Lominska C, Estes CF, Neupane PC, Shnayder Y, TenNapel MJ \& O'Neil MF. CASTLE thyroid tumor: a case report and literature review. Frontiers in Oncology 20177 207. (https://doi.org/10.3389/ fonc.2017.00207)

10 Nixon IJ, Coca-Pelaz A, Kaleva AI, Triantafyllou A, Angelos P, Owen RP, Rinaldo A, Shaha AR, Silver CE \& Ferlito A. Metastasis to the thyroid gland: a critical review. Annals of Surgical Oncology 2017 24 1533-1539. (https://doi.org/10.1245/s10434-016-5683-4)

Received in final form 25 April 2019

Accepted 15 May 2019 